\title{
Multicentric Musculoskeletal Hydatid Cysts - A Rare Case Report
}

\section{Prem James Charles ${ }^{1 *}$, Harshvardhan ${ }^{2}$ and Selvaraj Periyasamy ${ }^{3}$}

${ }^{1}$ Specialist Orthopedic Surgeon, Aster Specialist Centre for orthopedics and Physiotherapy, Dubai, UAE

${ }^{2}$ Specialist Orthopedic Surgeon, Aster Specialty Clinic, Discovery Gardens, Dubai, UAE

${ }^{3}$ Specialist Orthopedic Surgeon, RALS Clinic, Al Qusais, Dubai, UAE

*Corresponding Author: Prem James Charles, Specialist Orthopedic Surgeon, Aster Specialist Centre For orthopedics and Physiotherapy, Dubai, UAE.
Received: September 06, 2021

Published: September 28, 2021

(C) All rights are reserved by Prem James Charles., et al.

\begin{abstract}
Hydatid disease is one of most common parasitic diseases affecting humans. Though no organ is exception, the hydatid cysts preferably develop in the liver and the lungs. Though the musculoskeletal involvement is known to be rare, it should be considered in the differential diagnosis of soft tissue tumors.

We report an unusual case of musculoskeletal hydatid disease presenting in multiple locations over a period of four years duration in a 34-year-old male patient. The disease manifested with painful swellings, initially on the lateral aspect of right hip, then on the left hip and later in the left shoulder region. They were treated by incision and drainage/surgical excision in combination with anti-helminthic medication. Final functional outcomes were found satisfactory.
\end{abstract}

Keywords: Hydatid Cyst; Musculoskeletal Echinococcosis; Echinococcus granulosus

\section{Introduction}

Hydatid Disease (Cystic Echinococcosis) is a zoonosis caused by the tapeworm Echinococcus granulosus. In the life cycle of E. granulosus, humans are the intermediate hosts whereas the dogs act as definitive hosts. Man gets infected by accidental ingestion of eggs from the faeces of definitive host. The ingested eggs gain into the circulation after penetrating the intestinal mucosa and start developing the cystic lesions in the suitable anatomical sites [1].

Manifestation of symptoms depends on the size, location, organs involved, compressive effects on adjacent tissues, complications viz rupture, anaphylaxis etc. Since this is slow growing lesion, majority of them are understandably asymptomatic in the early stages.
Infection with E. granulosus results in development of solitary or multiple hydatid cysts; majority of which occurs in the liver $(70 \%)$ and in the lungs (20\%). Involvement of musculoskeletal tissues is uncommon ( $1 \%$ to $4 \%$ ) even in the endemic regions [2]. If involved, the disease has been frequently reported with pelvic, thigh, and paravertebral muscles [3].

In suspected cases, careful clinical history, physical examination along with serological, radiological and histopathological investigations are necessary for confirming the diagnosis.

Here, we present a case of primary intramuscular and subperiosteal hydatid cyst appeared in three different locations in a 34 -year-old male patient over a 4-year period of time. We also would like to discuss the pitfalls in diagnosis and management of this otherwise a rare entity. 


\section{Case History}

Site 1

In August 2016, a 30-year-old male presented with complaints of painful swelling on the lateral aspect of right hip region for four weeks duration. There was no history of trauma, fever, weight loss or any other constitutional symptoms. Clinical examination revealed diffusely tender, firm swelling measuring 00x00 $\mathrm{cm}$ over right the greater trochanter. Plain $\mathrm{x}$-ray (Figure $1 \mathrm{a}$ and $1 \mathrm{~b}$ ) showed calcification with no attachments on bony structures.

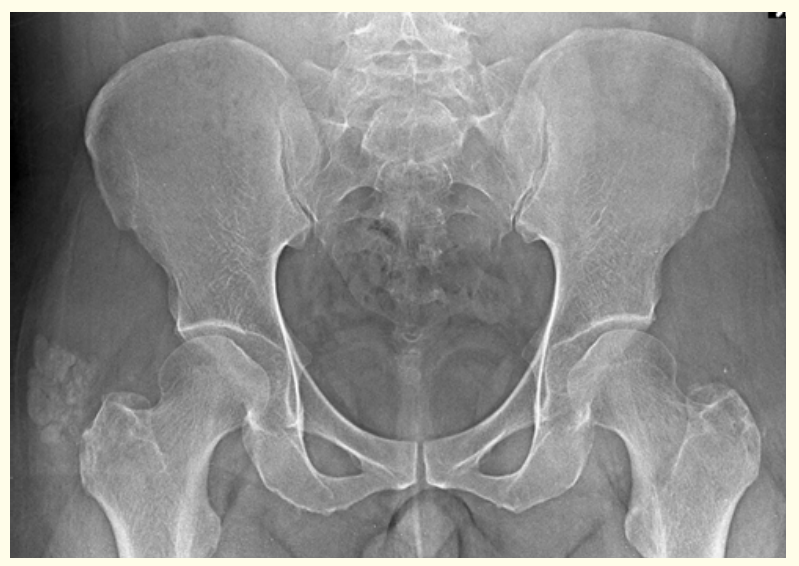

$1 a$

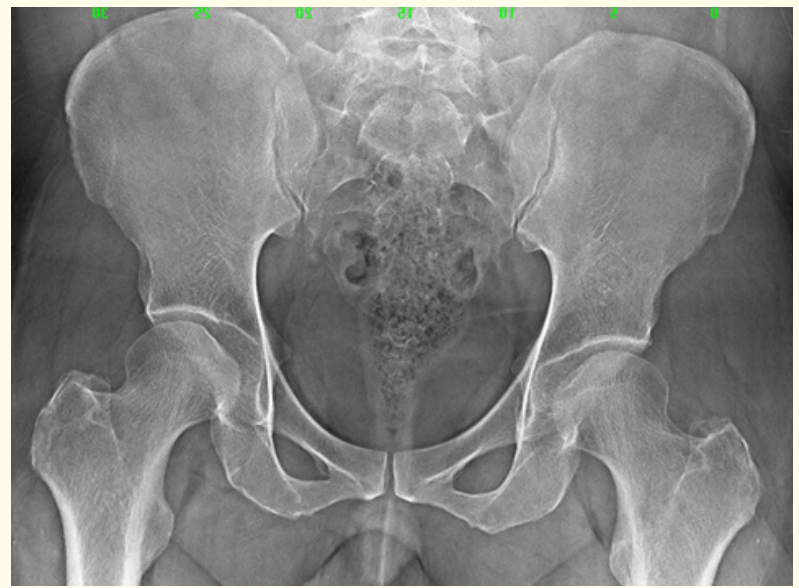

$1 b$

Figure 1a and 1b: Radiographs of right hip. Note the calcification near right greater trochanter. $1 \mathrm{a}$. Pre-op, 1b. Post-op.
As the presentation resembled soft-tissue mass on clinical and radiological examination, the patient was planned for an excision and biopsy. Upon the exploration, the gluteus medius was found necrosed; about $50 \mathrm{ml}$ of creamy thick fluid found collected under the deep fascia. The fluid was drained and the devitalized tissues were excised leaving a reasonably good margin of healthy tissues. Thorough saline irrigation was given. Scrapings and other tissues were sampled for biopsy. Diagnosis of echinococcus infection was made only after the histopathological examination (Figure 2a and $2 b)$. Anti-helminthic drug albendazole was given postoperatively for period of 3 weeks.

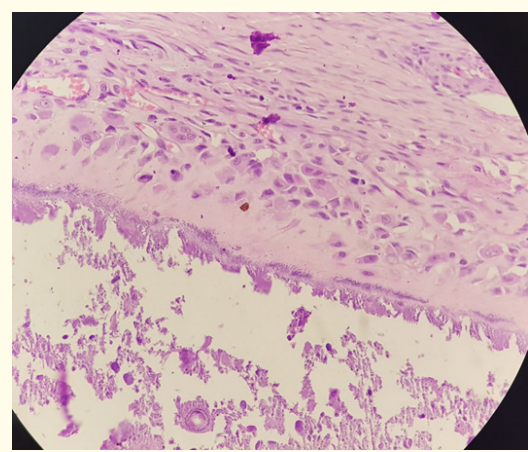

$2 a$

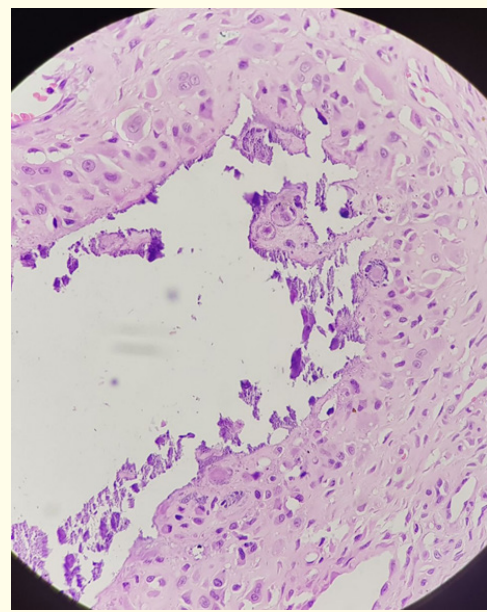

2b

Figure 2: Biopsy from right hip. 2a -The germinal layer, adventitia and calcified contents of the parasitic cyst. (400× H\&E). $2 b$-The germinal layer with scolex head, calcification and adjacent giant cell reaction $(400 \times \mathrm{H} \& \mathrm{E})$. 
Site 2

In November 2019, the same patient presented with almost similar complaints on the left hip region. No pain or swelling on the previously operated site, i.e. the right hip region. MRI investigation was carried out (Figure 3a and 3b).

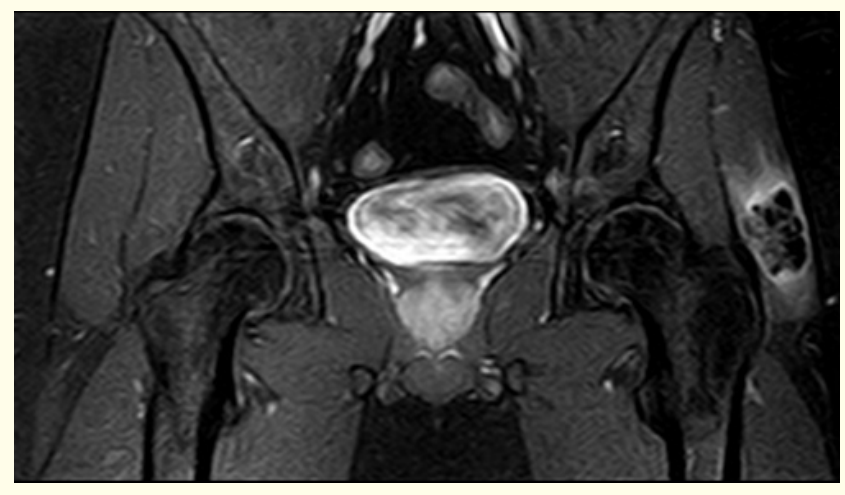

3a

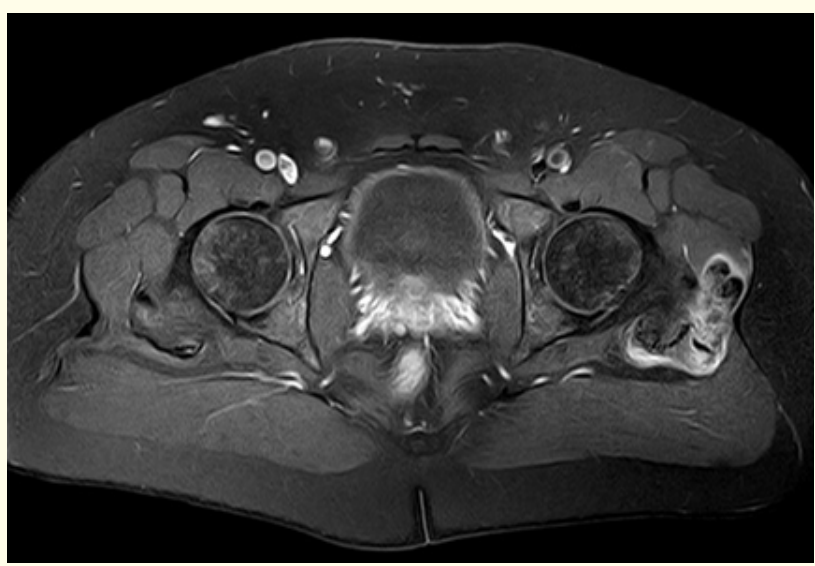

3b

Figure 3a and 3b: Magnetic resonance images of the left hip.

During the surgical procedure, about $5 \times 4 \times 4 \mathrm{~cm}$ sized cystic swelling was found on the anterolateral aspect, superficial to the gluteus medius muscle (Figure 4). Wide excision was performed followed by copious saline lavage. Biopsy confirmed the same tapeworm infection. Similar postoperative protocol was followed.

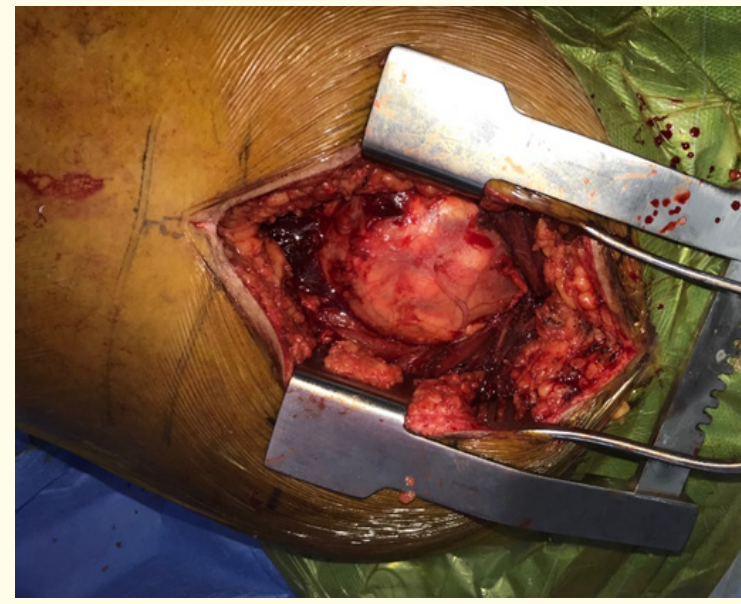

Figure 4: Per-op photo of the left hip.

Site 3

In March 2020, the above said patient reported with diffuse swelling about the left shoulder. MRI images showed three cystic masses, (measuring $16 \times 6 \times 5 \mathrm{~mm}, 11 \times 4 \times 3 \mathrm{~mm}$ and $10 \times 4 \times 5$ $\mathrm{mm}$ ) occupying at the tendinous portions of infraspinatus and subscapularis muscles (Figure 5). Additionally, a well-circumscribed cyst measuring $50 \times 45 \times 25 \mathrm{~mm}$ was seen eroding the cortex of lateral end of the clavicle, with reactive hyperostosis in the remaining part of the bone (Figure 6a and 6b).

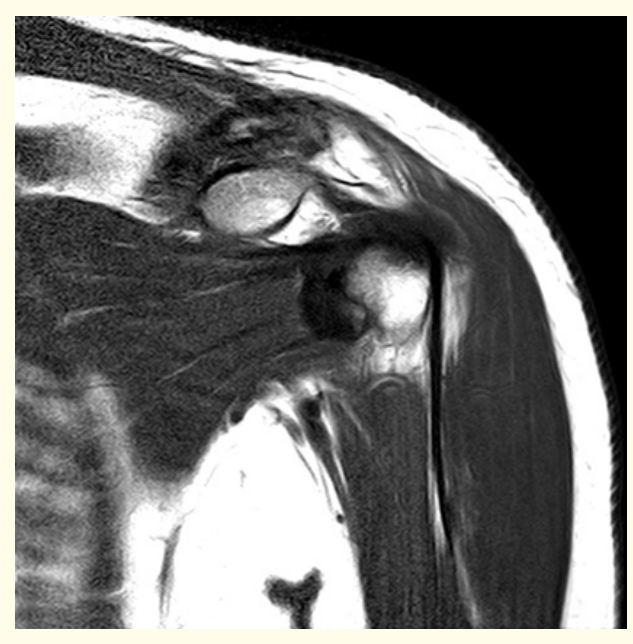




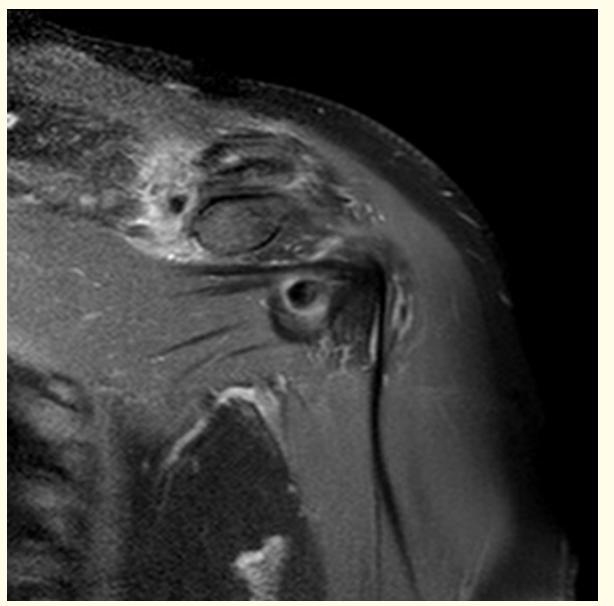

Figure 5: Magnetic resonance images of the left shoulder.

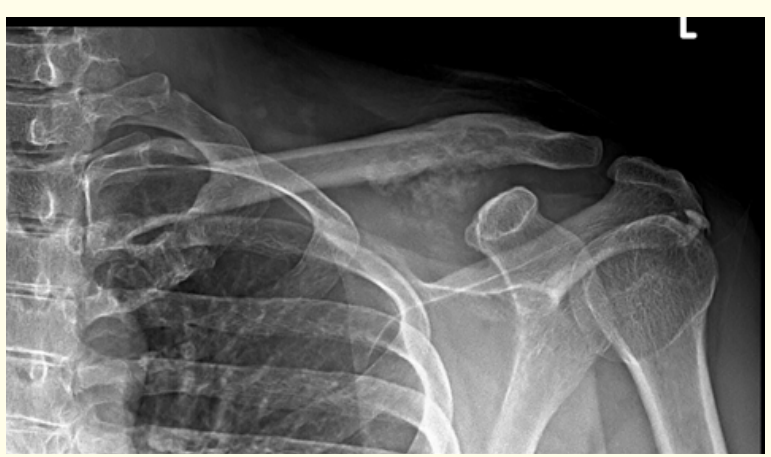

$6 a$

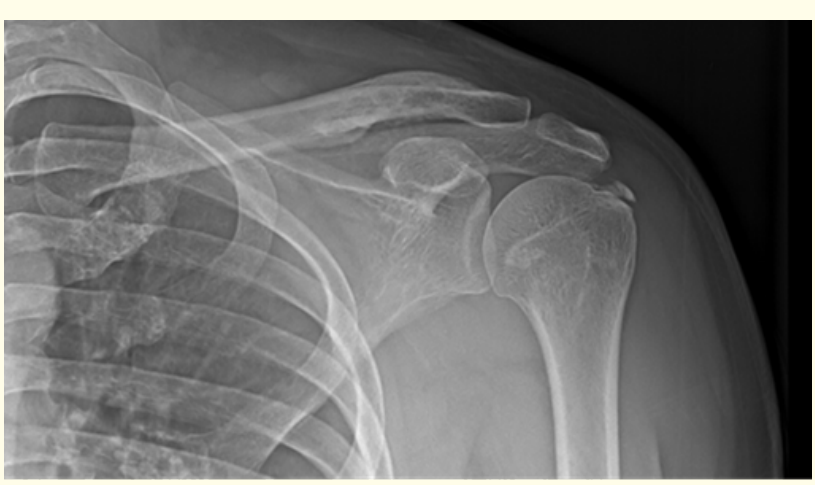

6b

Figure 6a and 6b: Radiographs of the left shoulder, 6a. Pre-op, 6b. Post-op.
Accessibility to all the aspects of cysts was met with difficulty. After considering multivesicular nature of the cysts and complex anatomy of the shoulder, percutaneous aspiration-injection-reaspiration (PAIR) technique was attempted and unsuccessful. Hence, the lesions were removed piecemeal through incisions made over the swellings. Saline wash and post operative medication were followed as before.

Among the three sites described here, in the first two (the hip regions), the cysts were confined to the intermuscular planes, whereas in the third (left shoulder), the pathology extended beyond the cortex of left clavicle. In all sites, we used hypertonic saline (the most widely used and easily available scolicidal agent) for irrigation to prevent recurrence or dissemination due to inadvertent intra-operative spillage.

\section{Discussion}

Hydatid cyst is a parasitic disease caused by Echinococcus granulosus, and is an endemic infection in Mediterranean countries, the Middle East, Africa, Asia, South America, and Australia [4]. Despite being a common zoonotic disease, the musculoskeletal involvement in the humans is rare [5].

Owing to its low prevalence in bone and muscle tissues and its similar insidious growth pattern with that of soft tissue tumors, definitive pre-operative diagnosis of muscular hydatid cysts poses great challenges to the clinicians. In fact, the most common symptom of isolated intramuscular hydatid cyst is a slow growing painless mass [6].

Many a times, the diagnosis is made either intraoperatively or after the histopathological results. We would like to remind here that, in our case during its first presentation, the diagnosis of hydatid cyst was made only after the surgery and confirmed by the biopsy report. When more than one muscle or its surrounding tissues are involved, as depicted in the left shoulder of this patient, encroachment of adjacent bones can happen [7].

Most studies show that the musculoskeletal hydatid cysts usually present as isolated swellings and are not seen to be associated with other organs involvement [8]. This was true in our patient as well, as he did not suffer any symptoms suggestive of internal organ involvements or space occupying lesions. 
Several serological tests like enzyme-linked immunosorbent assay (ELISA), Western blot, indirect hemagglutination antibody assays are available for the diagnosis of E. granulosus infection [9]. These tests show positivity over 90 percent cases of liver hydatid cysts, and less than 30 percent in musculoskeletal cysts [6].

Computed tomography (CT) gives information such as size, site, calcifications and number vesicles in the cysts. It also shows the relationship with the surrounding tissues [10].

MRI is the diagnostic modality of choice as for as the musculoskeletal hydatid cysts are concerned. It provides a better visualization regarding the extent of the lesion and its relations with adjacent neurovascular structures [11].

Although periosteal reaction, osteosclerosis or calcification are not specific for hydatid bone disease, when dealing with cases of chronic osteomyelitis, hydatid infection should be considered in differential diagnosis especially in the endemic regions [12].

Surgical removal of the cyst is the best treatment approach for surgically accessible muscular cysts [13]. Chemotherapy with albendazole remains the drug of choice both in pre and postoperative adjuvant therapy. Mebendazole and praziquantel are the other alternatives. The importance of antihelminthics intake lies on reducing the chances of metastatic infections and recurrences [14]. For inoperable cases, albendazole in combination with percutaneous aspiration-injection-reaspiration (PAIR) is considered a safe option [15].

\section{Limitations of the Study}

Relapses/recurrences are high if surgery is carried out without complete and effective antihelminthic coverage [16]. In addition to complete excision, duration of chemotherapy is considered a key factor in achieving effective eradication. Also, the responses to treatment will consume longer time to if the cysts are larger [17]. WHO suggests preoperative coverage for a period of 4 - 30 days [18].

We must have been aware of the above mentioned evidences and should have initiated antihelminthic agents prior to the procedure and properly monitored during the post-operative periods. The efficacy of albendazole increases if given for 3 months, but our case had taken only for 3 weeks which was a major (among the oth- ers) drawback in the management.

We accept that inadequate intake of albendazole and insufficient post-op monitoring might have contributed to successive development of cysts in this patient, albeit on different locations.

\section{Conclusion}

Primary musculoskeletal hydatid cyst is a rare condition. It requires high index of suspicion during the diagnosis of soft tissue swellings, particularly in the endemic areas. Definitive diagnosis requires a multidisciplinary approach which includes clinical, radiological and histological examinations. Surgical procedures combined with albendazole yields a better functional outcome. Most importantly, albendazole must be administered before and after the surgical procedures to avoid recurrence and secondary hydatidosis.

\section{Disclosures}

None.

\section{Conflict of Interest}

The authors declare that there is no conflict of interest regarding the publication of this manuscript.

\section{Funding Sources}

None.

\section{Bibliography}

1. Schantz PMK and P Brunetti. "Echinococcosis". In: Guerrant RLW, D.H.; Weller, P.F., editor. Tropical Infectious Diseases: Principles, Pathogens and Practice. (2 ${ }^{\text {nd }}$ edition), Churchill Livingstone (Elsevier), Philadelphia, USA (2006): 1304-1325.

2. Hamdi MF., et al. "Primary hydatid cyst of the biceps femoris". Musculoskeletal Surgery 94 (2010): 59-61.

3. A Lamine., et al. "Primary hydatidosis of the peripheral muscles:7 case reports”. Acta Orthopaedica Belgica 59 (1993): 184-188.

4. Kayaalp C., et al. "Primary subcutaneous hydatid cysts: a review of 22 cases". International Journal of Surgery 9 (2011): 117-121.

5. García-Alvarez F., et al. "Musculoskeletal hydatid disease: A report of 13cases”. Acta Orthopaedica Scandinavica 73.2 (2002): 227-231.

Citation: Prem James Charles., et al. "Multicentric Musculoskeletal Hydatid Cysts - A Rare Case Report". Acta Scientific Orthopaedics 4.10 (2021): $103-108$. 
6. Gougoulias NE., et al. "Skeletal muscle hydatid cysts presenting as soft tissue masses". Hippokratia 14.2 (2010): 126-130.

7. Ammari FF., et al. "Hydatid disease of the musculoskeletal system". Surgery 124.5 (1998): 934-937.

8. Drimousis PG., et al. "Unusual site of recurrent musculoskeletal hydatid cyst: Case report and brief review of the literature". World Journal of Gastroenterology 12.34 (2006): 5577-5578.

9. Yazar S and Altıntaș N. "Serodiagnosis of cystic echinococcosis in Turkey”. Helminthology 40 (2003): 9-13.

10. Sachar S., et al. "Uncommon locations and presentations of hydatid cyst". The Annals of Medical and Health Science Research 4.3 (2014): 447-452.

11. Arkun R and Mete BD. "Musculoskeletal hydatid disease". Musculoskeletal Radiology 15 (2011): 527-540.

12. Zlitni M., et al. "Hydatid cyst of bone: diagnosis and treatment". World Journal of Surgery 25 (2001): 75-82.

13. Madhar M., et al. "Primary hydatid cyst of the thigh: On seven cases". Journal of Musculoskeletal Surgery and Research 97 (2013): 77-79.

14. Siles-Lucas M., et al. "Progress in the pharmacological treatment of human cystic and alveolar echinococcosis: Compounds and therapeutic targets". PLOS Neglected Tropical Diseases 12.4 (2018): e0006422.

15. Hui M., et al. "Isolated musculoskeletal hydatid disease: diagnosis on fine needle aspiration and cell block". The Journal of Parasitic Diseases 39 (2015): 332-335.

16. Arazi M., et al. "Primary echinococcus infestation of the bone and muscles". Clinical Orthopaedics and Related Research 432 (2005): 234-241.

17. Todorov T., et al. "The types and timing of the degenerative changes seen in the cysts during and after benzimidazole treatment of cystic echinococcosis". Annals of Tropical Medicine and Parasitology 99.7 (2005): 649-659.

18. WHO Informal Working Group. International classification of ultrasound images in cystic echinococcosis for application in clinical and field epidemiological settings". Acta Tropica 85.2 (2003): 253-261.
Volume 4 Issue 10 October 2021

(C) All rights are reserved by Prem James Charles., et al. 